\title{
MS15-P10 | Squeezing the Most Data out of Your High-Pressure Experiment
}

Graf, Jürgen (Incoatec GmbH, Geesthacht, GER); Stürzer, Tobias (Bruker AXS GmbH, Karlsruhe, GER); Ott, Holger (Bruker AXS $\mathrm{GmbH}$, Karlsruhe, GER); Dera, Przemyslaw (University of Hawaii at Manoa, Honolulu, USA); Ruf, Michael (Bruker AXS Inc., Madison, USA); Radcliffe, Paul (Incoatec GmbH, Geesthacht, GER); Michaelsen, Carsten (Incoatec GmbH, Geesthacht, GER)

Within the last decade high pressure studies have received significant increase of interest. Present typical applications range from the investigation of high-pressure polymorphism of solid-state organics as part of the pharmaceutical drug development to the study of rocks and minerals with an applied pressure of up to $50 \mathrm{GPa}$.

A major challenge in the field of high pressure crystallography is the acquisition of data of sufficient quality and completeness for a successful structure determination. This presentation will be reviewing recent advances in hardware development, such as X-ray sources with radiation harder than Mo-K $\alpha$, and highlight the latest improvements in software, which help in tackling the problems with data acquisitions in high-pressure experiments.

Using a Bruker D8 VENTURE system, we will be demonstrating the advanced hardware capabilities and processing methods based on selected data, including data from the Indium METALET X-ray source. Further, we will be showing how to increase the completeness of high-pressure experiments by mounting multiple samples in a diamond anvil cell and measuring and processing data concurrently.

The high flexibility of the D8 VENTURE can be further expanded by adding enhanced features typically only available at synchrotron facilities, e.g. for studying tiny mineral crystals enclosed in diamond anvil cells. These features include highly accurate, motorized sample positioning and the ability to monitor the intensity of the X-ray beam passing through the diamond anvil cell, as well as an extension for online pressure measurements based on ruby fluorescence. This makes the D8 VENTURE system "a little synchrotron at home". 